\title{
Surgical septal myectomy outcome for obstructive hypertrophic cardiomyopathy after alcohol septal ablation
}

\author{
Qiulan Yang ${ }^{1}$, Changsheng Zhu ${ }^{1}$, Hao Cui ${ }^{2}$, Bing Tang ${ }^{3}$, Shengwei Wang ${ }^{3}$, Qinjun Yu ${ }^{4}$, Shihua Zhao ${ }^{5}$, \\ Yunhu Song ${ }^{1}$, Shuiyun Wang ${ }^{1}$ \\ ${ }^{1}$ Department of Cardiovascular Surgery, Fuwai Hospital, National Center for Cardiovascular Diseases, Chinese Academy of Medical Sciences and \\ Peking Union Medical College, Beijing, China; ${ }^{2}$ Department of Cardiovascular Surgery, Mayo Clinic, Rochester, MI, USA; ${ }^{3}$ Department of Cardiac \\ Surgery, Beijing Anzhen Hospital, Capital Medical University \& Beijing Institute of Heart, Beijing, China; ${ }^{4}$ Department of Anesthesiology, Fuwai \\ Hospital, National Center for Cardiovascular Diseases, Chinese Academy of Medical Sciences and Peking Union Medical College, Beijing, China; \\ ${ }^{5}$ Department of Magnetic Resonance Imaging, Fuwai Hospital, National Center for Cardiovascular Diseases, Chinese Academy of Medical Sciences \\ and Peking Union Medical College, Beijing, China \\ Contributions: (I) Conception and design: S Wang; (II) Administrative support: C Zhu, H Cui, B Tang, S Wang, S Zhao; (III) Provision of study \\ materials or patients: C Zhu; (IV) Collection and assembly of data: Q Yang; (V) Data analysis and interpretation: C Zhu, H Cui, B Tang, S Wang, S \\ Zhao; (VI) Manuscript writing: All authors; (VII) Final approval of manuscript: All authors. \\ Correspondence to: Shuiyun Wang. Department of Cardiovascular Surgery, Fuwai Hospital, National Center for Cardiovascular Diseases, Chinese \\ Academy of Medical Sciences and Peking Union Medical College, Beilishi Road 167, Xicheng District, Beijing 100037, China. Email: wsymd@sina.com.
}

Background: Although surgical treatment of residual obstruction after alcohol septal ablation (ASA) is often challenging in patients with obstructive hypertrophic cardiomyopathy (OHCM) there are very few relevant clinical reports. Thus, outcomes of surgical septal myectomy (SSM) in this subgroup of patients remain to be determined. Therefore, this study aimed to determine the surgical and follow-up outcomes in patients with OHCM exhibiting residual obstruction after ASA.

Methods: We collected case data for 62 patients with OHCM and residual obstruction after ASA who underwent SSM at Fuwai Hospital between January 2002 and June 2019. Propensity score matching with patients having had a myectomy as the only invasive procedure-was conducted in a 1:2 ratio. Echocardiography parameters, surgery results, and follow-up outcomes were compared between the groups.

Results: The prior ASA group had a higher incidence of complete atrioventricular block (AVB) and subsequently postoperative permanent pacemaker (PPM) implantation than the primary myectomy group (9.7\% vs. $1.6 \%, \mathrm{P}=0.01$ ). Two patients died within 30 days after surgery in the prior ASA group, and one patient died in the primary myectomy group, with an operative mortality rate of $3.2 \%$ and $0.8 \%$, respectively $(\mathrm{P}=0.2)$. The 5 -year event-free survival rate was $86.0 \%$ in the prior ASA group (median follow-up period: 3.2 years; mean: $3.9 \pm 2.6$ years; maximum, 10.6 years) and $88.5 \%$ in the primary myectomy group (median follow-up period: 2.4 years; mean $2.8 \pm 1.7$ years; maximum, 9.1 years) $(\mathrm{P}=0.2)$. During follow-up, four of $62(6.5 \%)$ patients in the prior ASA group and one of $124(0.8 \%)$ patients in the primary myectomy group progressed to advanced heart failure $(\mathrm{P}=0.025)$.

Conclusions: Patients with OHCM following ASA are at an increased risk of developing AVB after SSM. Their surgical outcomes, and long-term survival rate were satisfactory and, osimilar to those for patients having had a myectomy as the only invasive procedure. In addition, they had an increased risk of advanced heart failure after SSM in the present study.

Keywords: Obstructive hypertrophic cardiomyopathy (OHCM); surgical septal myectomy (SSM); alcohol septal ablation (ASA); atrioventricular block (AVB)

^ ORCID: 0000-0003-4506-2179.

(c) Journal of Thoracic Disease. All rights reserved. 
Submitted Oct 02, 2020. Accepted for publication Dec 17, 2020.

doi: $10.21037 /$ jtd-20-2779

View this article at: http://dx.doi.org/10.21037/jtd-20-2779

\section{Introduction}

Hypertrophic cardiomyopathy (HCM) is characterized by asymmetric thickening of the ventricular septum and affects approximately one in 500 people in the general population (1). Obstructive hypertrophic cardiomyopathy (OHCM) is a severe type of HCM defined as a left ventricular outflow tract (LVOT) peak pressure gradient of $\geq 30 \mathrm{mmHg}$ at rest or after provocation (1).

Surgical septal myectomy (SSM) and alcohol septal ablation (ASA) are invasive ventricular septal reduction procedures for the treatment of OHCM (2). They can effectively relieve obstruction and symptoms in patients in whom the optimal drug therapy has failed. SSM has been established as the gold standard for the treatment of patients with drug-refractory OHCM (3). ASA, a less invasive procedure, has recently become an alternative treatment for drug-refractory OHCM (4). However, some patients with $\mathrm{OHCM}$ who have undergone ASA may require subsequent SSM due to drug-refractory recurrent symptoms caused by residual obstruction (5). Notably, relevant clinical reports concerning the surgical treatment of residual obstruction post-ASA, which is often more challenging, are limited. Therefore, this study aimed to examine the surgical outcomes in cases of residual obstruction post-ASA and follow-up outcomes of SSM in this subgroup of patients with OHCM. We present the following article in accordance with the STROBE reporting checklist (available at http:// dx.doi.org/10.21037/jtd-20-2779).

\section{Methods}

\section{Participants}

The study was conducted in accordance with the Declaration of Helsinki (as revised in 2013). The study was approved by The Institutional Review Board of Fuwai Hospital (NO.2020-1315) and informed consent was taken from all the patients. The study included 62 patients with $\mathrm{OHCM}$ who had undergone ASA and subsequent SSM in our hospital between January 2002 and June 2019 due to residual LVOT obstruction and the presence of recurring symptoms refractory to drug therapy. Nineteen patients previously underwent ASA at our institution, and 43 patients underwent septal ablation elsewhere. During this period, patients with prior ASA were matched to patients who had undergone septal myectomy as the only procedure using a one-to-two propensity score matching method based on sex, age, preoperative New York Heart Association function class, maximum septal thickness and peak LVOT gradient. Patients with previous cardiac surgery were excluded. Informed consent was taken from all the patients. Figure 1 shows the flow chart of the study.

\section{Data collection}

Medical records, physical examination results, imaging results, surgical procedure, postoperative outcomes, and operative mortality rates (defined as death before discharge or within 30 days after surgery) were collected for all patients.

\section{Surgical technique}

We resected the hypertrophied ventricular septum by performing a modified procedure, which evolved from the classic Morrow operation (6). Concomitant surgery was performed when necessary. If intraoperative transesophageal echocardiography showed that the peak LVOT pressure gradient was still $>30 \mathrm{mmHg}$ or systolic anterior motion of the anterior mitral leaflet existed, cardiopulmonary bypass was resumed for further resection.

\section{Follow-up}

The most recent follow-up data were obtained through subsequent outpatient visits and telephone interviews with patients or their family members. Follow-up hard endpoints included all-cause death and sudden death, and the composite endpoint included all-cause death, successful resuscitation, heart failure requiring hospitalization, new-onset atrial fibrillation, documented stroke beyond 30 days after surgery, and permanent pacemaker (PPM) 


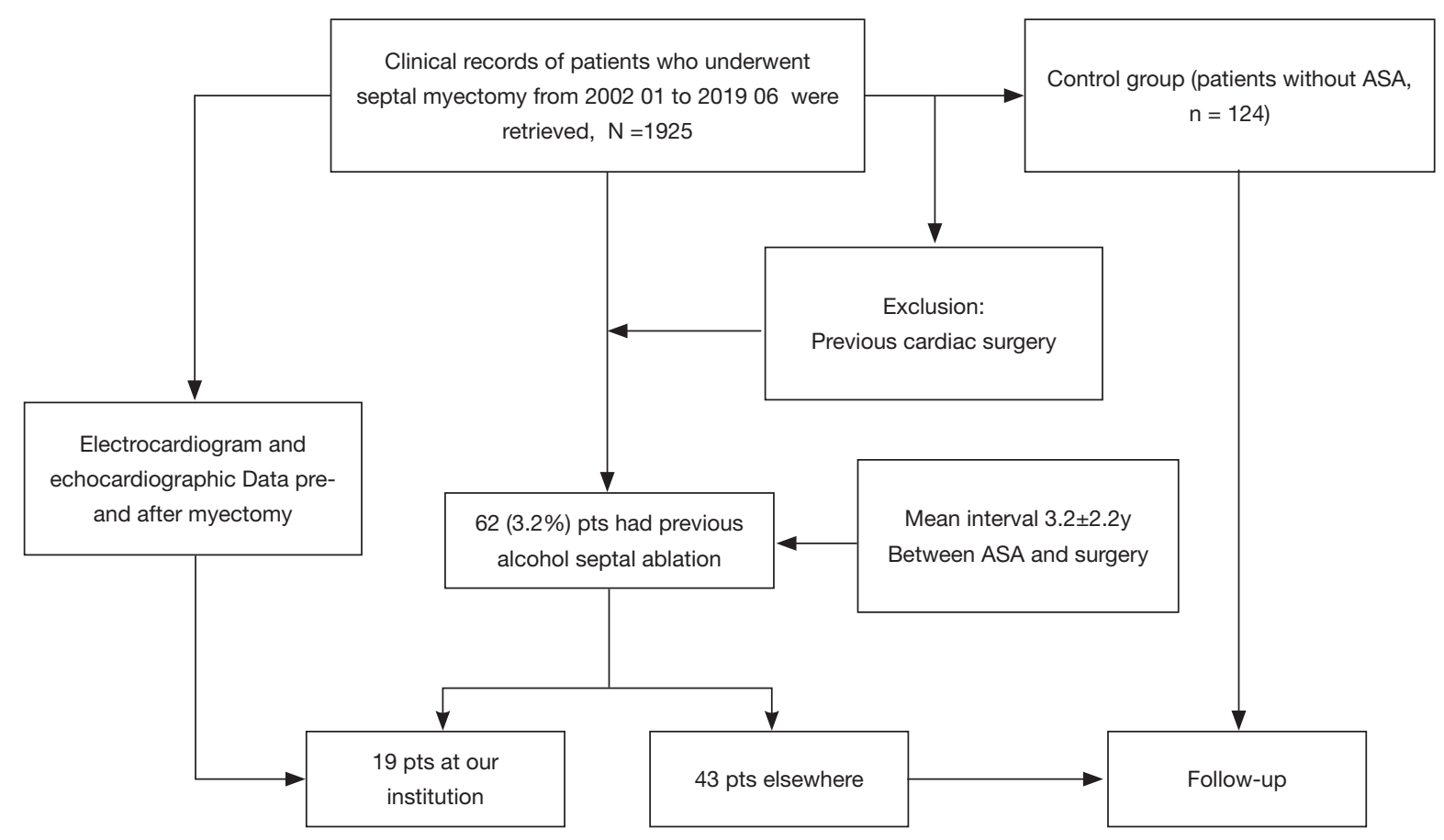

Figure 1 The flow chart of the study.

implantation beyond 30 days after surgery. The first event was used when a patient experienced with multiple events. All-cause death was defined as death from any cause, including operative mortality. Successful cardiopulmonary resuscitation was considered equivalent to sudden death. Stroke was defined as transient or permanent neurological dysfunction and disability from vascular causes, including transient ischemic attacks lasting less than 24 hours.

\section{Statistical analysis}

Categorical variables are expressed as frequencies and percentages, and continuous variables are expressed as the mean \pm standard deviation or median. The chi-square test or Fisher's exact test was used to compare categorical variables. The two-sample $t$-test was used to compare continuous variables, and the paired $t$-test was used for pairwise comparisons. Kaplan-Meier curves were used to estimate survival rates. Death before hospital discharge or within 30 days after surgery was included in survival analyse. Statistical significance was defined as a two-sided $\mathrm{P}$ value of less than 0.05 . All statistical analyses were performed using SPSS version 22.0 (SPSS Inc., IBM Company, Chicago,
IL, USA) and MedCalc, version 15.0 (MedCalc Software, Ostend, Belgium).

\section{Results}

\section{Baseline and surgical parameters}

This study enrolled 62 patients with OHCM (women, 18) who had undergone ASA and subsequent SSM at our hospital. The average interval between the last ASA and SSM was $3.2 \pm 2.9$ years, and the minimum interval was 1 month. The control group included 124 patients with OHCM (women 32), who had undergone septal myectomy as the only procedure. Baseline data are summarized in Table 1.

\section{Potential causes of ASA failure}

Forty-four patients (71\%) underwent cardiac magnetic resonance $(\mathrm{CMR})$ imaging before surgery. Their CMR images were retrieved and re-read to identify potential causes of ASA failure. The infarcted myocardium caused by ASA had diverse locations and extents. In 25 patients $(56.8 \%)$, the infarct was located in the hypertrophic area, but the infarcted area was smaller and was located in the 
Table 1 Baseline data

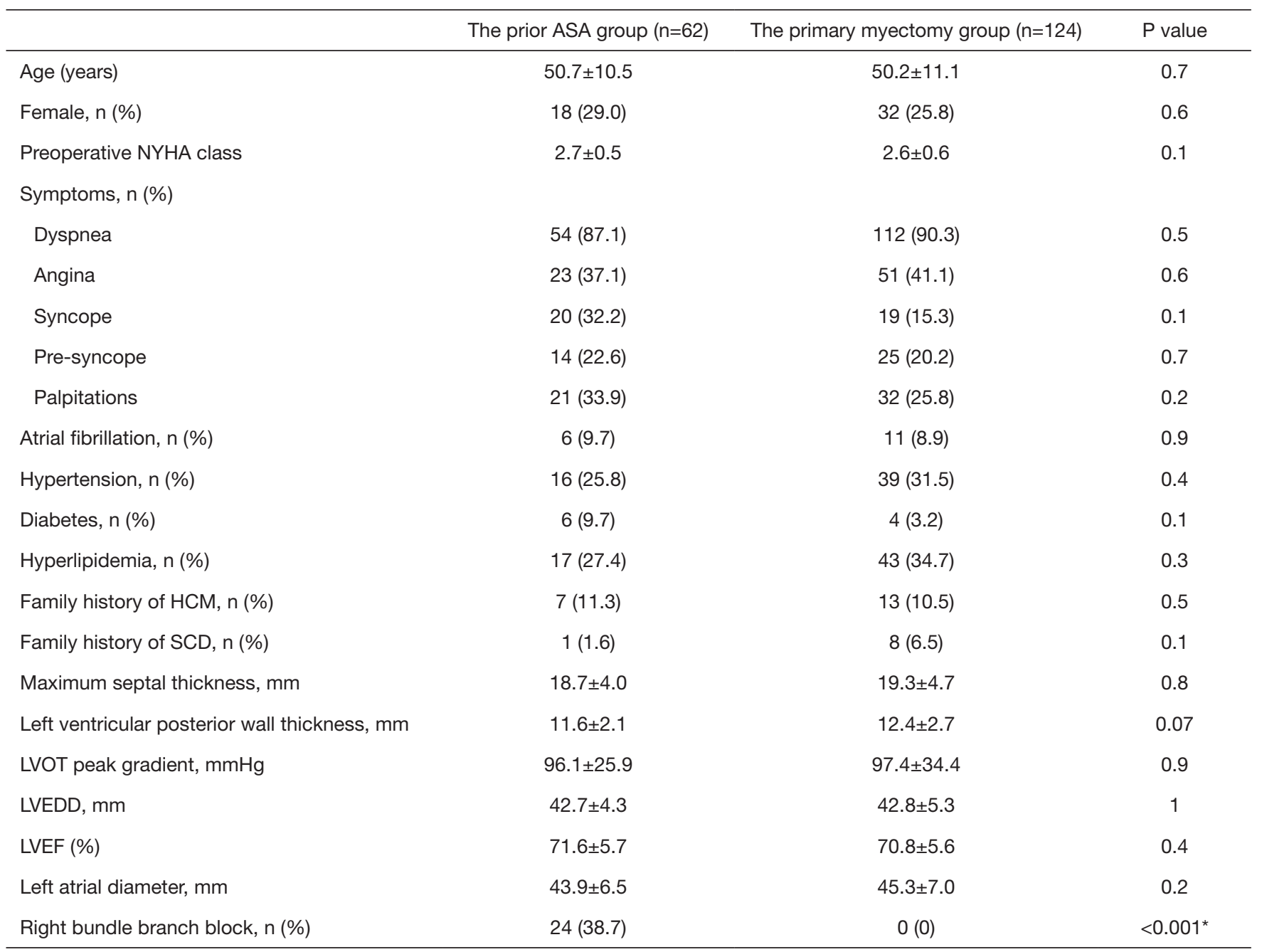

ASA, alcohol septal ablation; NYHA, New York Heart Association; HCM, hypertrophic cardiomyopathy; SCD, sudden cardiac death; LVOT, left ventricular outflow tract; LVEDD, left ventricular end-diastolic dimension, LVEF, left ventricular ejection fraction.

lower part of the hypertrophic area. In another 15 patients (24.2\%), the infarction caused by ASA was outside the target for the ablated hypertrophic area. In the remaining four patients, no ablation scarring was observed.

\section{PPM implantation}

In the prior ASA group, 24 patients (38.7\%) had preoperative right bundle branch block (RBBB), of whom $6(9.7 \%)$ developed postoperative left bundle branch block after surgery and eventually progressed into complete atrioventricular block (AVB). All six patients had a PPM implanted before discharge. Notably, 3 patients $(4.8 \%)$ with RBBB had postoperative left anterior branch block, and they are at risk of developing complete AVB in the future. During the follow-up period, a 50-year-old male patient (1.6\%) required PPM implantation 33 days after discharge.

In the control group, no patient had preoperative RBBB. Two patients (1.6\%) had AVB and then underwent PPM implantation early after surgery. The incidence of complete AVB and subsequent postoperative PPM implantation was higher in the prior ASA group than in the control group (9.7\% vs. $1.6 \%, \mathrm{P}=0.01$; Table 2 ).

\section{Surgical outcomes}

The echocardiographic parameters of the two patient groups significantly improved after surgery (Table 3). 
Table 2 Summary of the surgical parameters of the study subjects

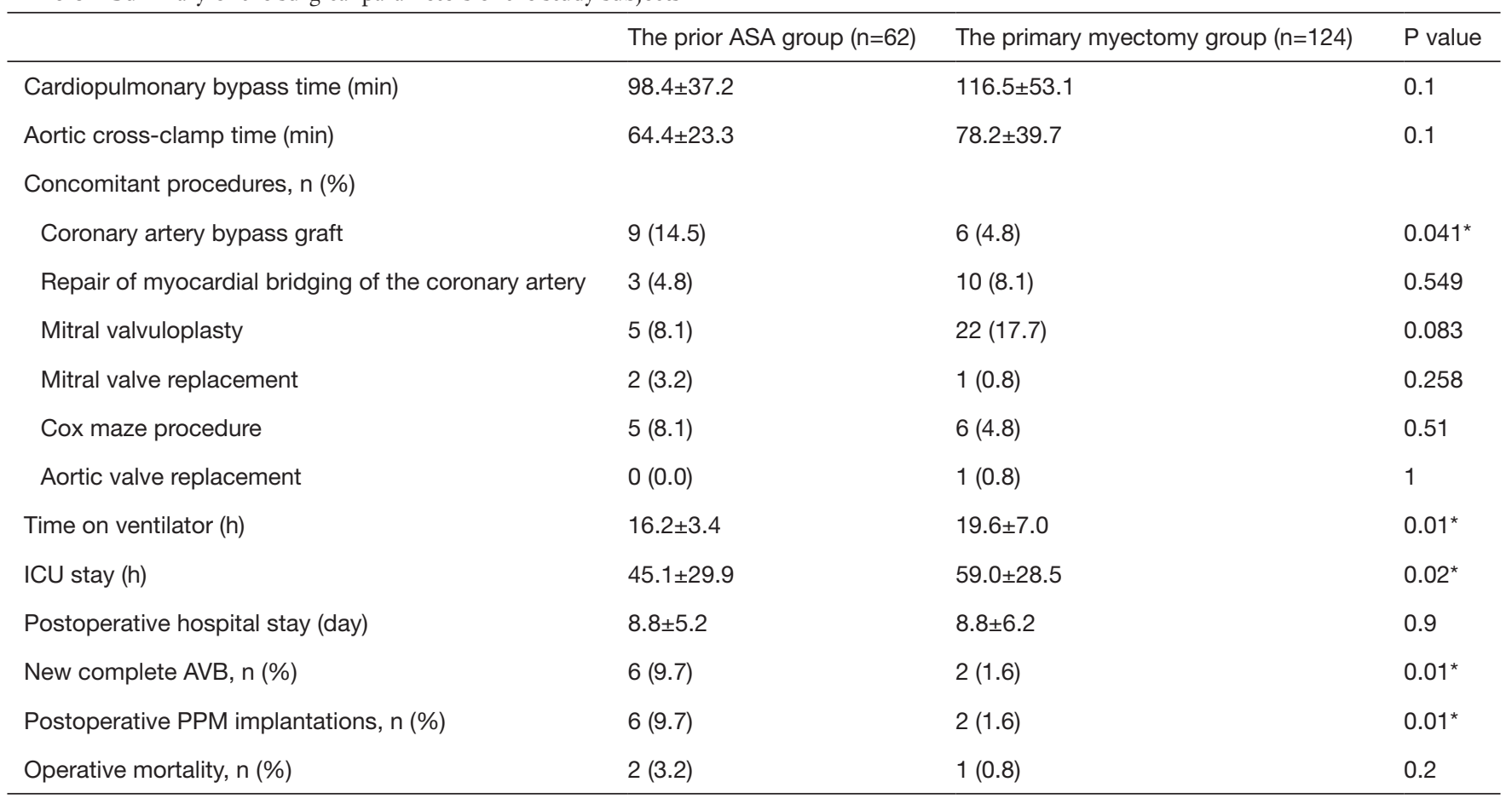

Values are presented as the means $\pm \mathrm{SD}$, medians $(\mathrm{IQR})$ or $\mathrm{n}(\%) .{ }^{*}, \mathrm{P}<0.05$ versus the prior ASA group. ASA, alcohol septal ablation; ICU, intensive care unit; AVB, atrioventricular block; PPM, permanent pacemaker.

Table 3 Preoperative and postoperative echocardiographic parameters

\begin{tabular}{|c|c|c|c|c|c|c|}
\hline & \multicolumn{3}{|c|}{ The prior ASA group $(n=62)$} & \multicolumn{3}{|c|}{ The comparison group $(n=124)$} \\
\hline Interventricular septal thickness, mm & $18.7 \pm 4.0$ & $13.9 \pm 4.2$ & $<0.001^{*}$ & $19.3 \pm 4.7$ & $13.3 \pm 3.3$ & $<0.001^{*}$ \\
\hline Posterior wall thickness, mm & $11.6 \pm 2.1$ & $11.0 \pm 1.7$ & 0.42 & $12.4 \pm 2.7$ & $11.3 \pm 2.4$ & $<0.001^{*}$ \\
\hline Maximal LVOT gradient, mmHg & $96.1 \pm 25.9$ & $13.5 \pm 6.6$ & $<0.001^{*}$ & $97.4 \pm 34.4$ & $13.3 \pm 6.7$ & $<0.001^{*}$ \\
\hline Left ventricular ejection fraction (\%) & $71.6 \pm 5.7$ & $63.5 \pm 6.7$ & $<0.001^{*}$ & $70.8 \pm 5.6$ & $62.0 \pm 5.2$ & $<0.001^{*}$ \\
\hline Left atrial diameter, mm & $43.9 \pm 6.5$ & $37.4 \pm 6.2$ & $<0.001^{*}$ & $45.3 \pm 7.0$ & $37.6 \pm 6.0$ & $<0.001^{*}$ \\
\hline Mitral regurgitation (0-5) & $2.4 \pm 1.0$ & $0.7 \pm 0.5$ & $<0.001^{*}$ & $2.2 \pm 1.1$ & $0.5 \pm 0.4$ & $<0.001^{*}$ \\
\hline
\end{tabular}

Values are presented as the means $\pm \mathrm{SD}$. ${ }^{*}, \mathrm{P}<0.05$ versus the prior ASA group. Mitral valve regurgitation was defined as: 0 , none; 1 , little or mild; 2, moderate; 3, moderate to severe; 4, severe. ASA, alcohol septal ablation; LVOT, left ventricular outflow tract.

\section{Operative mortality}

In the ASA group, two patients (3.2\%) died after surgery. In one patient, a perforation of the ventricular septum was found on transesophageal echocardiography during operation, and the perforation was caused by necrotic septal tissues after alcohol ablation. The patient was returned to the recovery room after repairing the perforation with polyester patch. The patient eventually died due to low cardiac output syndrome and multiple organ failure on postoperative day 11. Another patient died due to cardiac output syndrome on postoperative day 28 . 


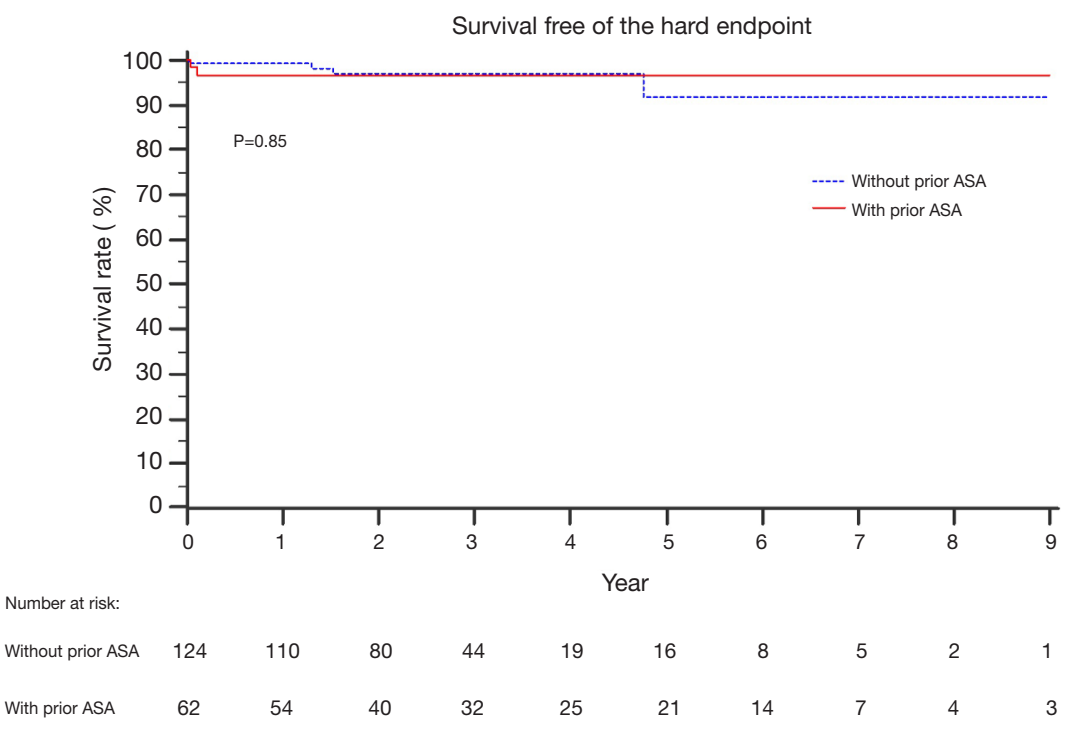

Figure 2 Survival rate curve in the present study.

Table 4 Clinical events beyond 30 days after surgery and at follow-up

\begin{tabular}{|c|c|c|c|}
\hline & \multicolumn{2}{|c|}{ Late (>30 days after myectomy) clinical events, $\mathrm{n}(\%)$} & $P$ value \\
\hline Secondary composite endpoint & $7(11.3)^{*}$ & $7(5.6)$ & 0.17 \\
\hline Permanent pacemaker implantation & $1(1.6)$ & $0(0.0)$ & 0.16 \\
\hline New-onset atrial fibrillation & $3(4.8)$ & $6(4.8)$ & 1 \\
\hline Stroke & $0(0.0)$ & $2(1.6)$ & 0.32 \\
\hline
\end{tabular}

*, one person had heart failure and atrial fibrillation concurrently.

In the control group, one patient $(0.8 \%)$ died within 30 days after surgery. There was no difference in operative mortality between the two groups $(\mathrm{P}=0.2$; Table 2$)$.

\section{Follow-up}

No patients were lost during follow-up. The median followup time in the prior ASA group was 3.2 years [interquartile range (IQR): $1.6-5.8$ years; mean: $3.9 \pm 2.6$ years]. No patients died during the follow-up period. The 5 -year hard endpoint-free survival rate was $96.8 \%$. In the primary myectomy group, the median follow-up time was 2.4 years (IQR 1.6-3.6; mean 2.8 \pm 1.7 ), and three patients died during the follow-up period, with a 5 -year hard endpoint-free survival rate of $91.7 \%$ (Figure 2). In the ASA group, three patients $(4.8 \%)$ without preoperative atrial fibrillation developed new-onset atrial fibrillation, of which one patient also experienced chronic heart failure (CHF) and received drug treatment. The other two patients underwent catheter ablation or received anticoagulation therapy. Except for one case of atrial fibrillation complicated by CHF, another three patients $(4.8 \%)$ developed $\mathrm{CHF}$ that required hospitalization. There were no stroke events during the follow-up period (Table 4).

At the follow-up period, four of $62(6.5 \%)$ patients and one of $124(0.8 \%)$ patients in the prior ASA group and the primary myectomy groups had progressed to advanced heart failure, respectively $(\mathrm{P}=0.025)$.

\section{Survival free of the composite endpoint}

The 5-year composite event-free survival rate was $86.0 \%$ in the prior ASA group and $88.5 \%$ in the primary myectomy group $(\mathrm{P}=0.19)$ (Figure 3). 


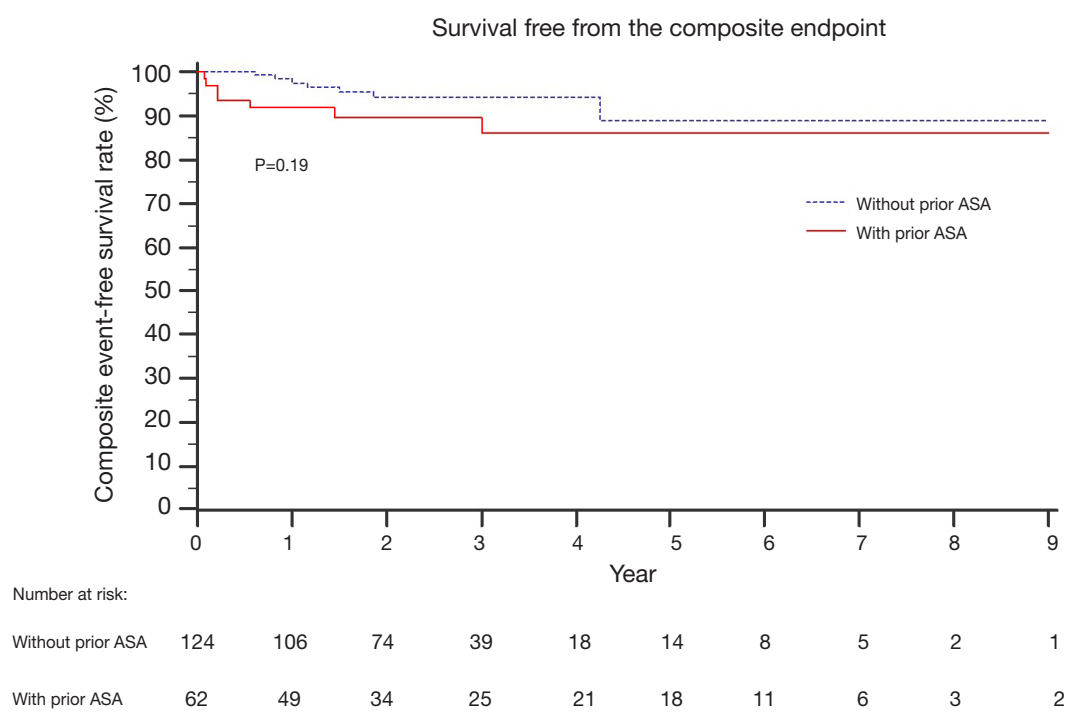

Figure 3 Survival free of composite endpoint.

\section{Discussion}

The results of this study indicate that SSM is a safe and effective method for treating residual LVOT obstruction in patients with post-ASA OHCM. In addition, since these patients are at risk for RBBB, our findings indicate that the risk of AVB increases after SSM.

ASA is a common non-drug therapy for OHCM that involves the injection of ethanol into the septal branch of the coronary artery to cause local infarction and regional remodeling of the hypertrophic myocardium in the basal segment of the ventricular septum, thereby widening the inner diameter of the LVOT, reducing the pressure gradient, and relieving obstruction. Moreover, the results of a small number of CMR images obtained suggest that inconsistency of the septal artery blood supply and hypertrophic obstruction in the ablation target zone may cause recurrent or residual symptomatic LVOT obstruction post-ASA $(7,8)$. In addition, other non-injected septal branches and circumflex branches may supply the ischemic interventricular septum and form collateral circulation. Furthermore, LVOT obstruction due in part to an abnormal mitral valve apparatus cannot be resolved by ASA. These characteristics limit the applicability of ASA in patients with OHCM.

With respect to endpoint events, this study showed that patients with OHCM had pathologic features in the ablation zone with interstitial fibrosis, myocardial necrosis, and transmural myocardial scaring post-ASA, which makes the subsequent SSM more challenging to some extent. Previously, Quintana et al. of the Mayo Clinic (9) reported 31 cases of patients with post-ASA OHCM undergoing SSM. In their series, two patients (6.5\%) died during the early postoperative period, and no patients died in the primary myectomy group, allowing the authors to conclude that post-ASA SSM increases the risk of cardiac death. In our study, two patients (3.2\%) died within 30 days after surgery. Numerically, mortality was higher in the post-ASA surgical myectomy group than in the control myectomy group, although no statistical significance was observed. Although mitral valve surgery was performed more frequently in the control myectomy group, which resulted in longer bypass and cross-clamp time, no statistical significance was observed, and mortality rates remained similar between the groups. In the prior ASA group, operative mortality and 5-year event-free survival rates were not inferior to those in the control myectomy group ( $\mathrm{P}=0.2$ and $\mathrm{P}=0.19$ respectively). This suggests that SSM post-ASA may not increase mortality risk in a dedicated HCM center in the contemporary era. Our study also demonstrated that advanced heart failure may be more likely to occur during the postoperative follow-up period in patients with OHCM and previous ASA. In terms of heart failure, this conclusion 
is similar to that reported by Quintana et al. Interstitial fibrosis and myocardial infarction leading to diastolic impairment may contribute to an increased risk of advanced heart failure (9). Thus, indications for septal reduction therapy should be strictly mastered. Myocardial contrast echocardiography (MCE) is recommended for accurate preoperative assessment of whether the distribution of the septal branch allows for ethanol delivery to the site of the hypertrophic obstruction, thereby preventing residual obstruction after ASA (10-12).

The results of this study show that SSM effectively relieved residual LVOT obstructions in patients with OHCM who had undergone ASA. Surgical corrective efficacy was obvious, and event-free survival rates (including new-onset atrial fibrillation, CHF requiring hospitalization, and stroke) were similar between the prior ASA and control myectomy groups. Although residual obstruction post-ASA makes SSM technically challenging, this study shows that the medium- and long-term followup outcomes of subsequent SSM for patients with post-ASA OHCM exhibiting residual obstruction were satisfactory at experienced treatment centers.

The results of this study suggest that 24 of the 62 patients with post-ASA OHCM (38.7\%) exhibited $\mathrm{RBBB}$, of whom seven patients (11.3\%) required PPM implantation after SSM. This rate was higher than that in the control group $(\mathrm{P}=0.01)$ and higher than that in previous studies in which SSM was the first choice for treatment $(13,14)$. A previous single-center retrospective cohort study included 375 patients who underwent ASA, of whom $20(5 \%)$ subsequently underwent SSM due to recurrent symptomatic LVOT obstruction (15). No patient died after the second surgery, and postoperative LVOT obstruction and clinical symptoms were significantly improved, but $12 \%$ of the patients required PPM implantation (2/17, 10\%; three patients had PPM implantation post-ASA). These postoperative AVB findings are similar to our findings. This suggests that, after the failure of previous ASA, clinicians should not only strive for anatomical correction of LVOT obstruction, but should also monitor patients for AVB after subsequent surgical treatment.

Reliable research evidence shows that the pressure gradient in the LVOT is significantly reduced after SSM, and 10-year survival rates are similar to those observed in an age- and gender-matched general population $(4,16)$. Thus, surgical myectomy has been established as the gold standard for children and adults with both $\mathrm{LV}$ outflow obstruction and severe drug-refractory symptoms (16). Given the characteristics of the surgical resection area, SSM is prone to damaging the left bundle branch. A retrospective analysis of 2,482 patients who underwent SSM at the Mayo Medical Center in the United States (13) showed a postoperative complete left bundle branch block rate of $38.8 \%$. However, this factor does not affect the long-term survival rate. Some researchers believe that the alleviation of LVOT obstruction is achieved by the change in the left ventricular contraction sequence caused by conduction damage (17). In addition, the present study revealed that the risk of AVB after SSM alone is low, but concomitant preoperative RBBB increases the risk of AVB after surgery $(13,18)$, and postoperative PPM affects long-term survival (13). Previous reports of ASA for the treatment of OHCM showed that ASA is associated with right bundle branch injury (19), with an incidence rate as high as $58 \%(8,20,21)$. This may be due to the injection of ethanol via the septal branch of the left anterior descending branch of the coronary artery, which also affects the blood supply to the right bundle branch and the left anterior branch. In addition, $8-22 \%$ of patients will have AVB requiring pacemaker implantation after alcohol ablation (2224). Therefore, clinicians utilizing ASA for the treatment of OHCM should be cautious of conduction system injury, and patient selection should be optimized. During the ASA procedure, a single rapid injection of ethanol is recommended, the amounts of ethanol use for ASA should be appropriate, MCE technology should be used to select correct septal branch, and injection through multiple ventricular septal arteries should be avoided to prevent permanent iatrogenic damage to the conduction system $(22,25)$.

A series of observational studies have compared the relative safety and efficacy of ASA and SSM. The results show that both treatments can relieve LVOT obstruction and improve New York Heart Association (NYHA) class in patients with $\mathrm{OHCM}$, but there may be differences in some complications and outcomes (20,22,26-31). A systematic review and meta-analysis showed that PPM implantation and re-intervention rates after SSM were significantly reduced when compared with post-ASA rates (22). For patients with OHCM with LVOT pressure gradients $\geq 50 \mathrm{mmHg}$, NYHA class III-IV, and significant left ventricular hypertrophy (>30 mm) (32), SSM is recommended as the initial treatment, while ASA is recommended as an alternative treatment $(14,33)$.

\section{Limitation}

This study has some limitations. The study was a retrospective cohort analysis with a small sample. 
Therefore, the conclusions must be confirmed in a largesample cohort study.

\section{Conclusions}

This study reported the early, medium-, and long-term outcomes of the largest group of patients with post-ASA OHCM who underwent SSM to date, and the results were satisfactory. Patients with OHCM are at risk for RBBB following ASA, leading to an increase in the incidence of AVB after SSM.

\section{Acknowledgments}

Funding: This study was supported by Clinical and Translational Research Fund of Chinese Academy of Medical Sciences (Grant Number: 2019XK320054), and Capital's Funds for Health Major Project of the Beijing Municipal Commission of Health (Grant Number: 2020-24036).

\section{Footnote}

Reporting Checklist: The authors have completed the STROBE reporting checklist. Available at http://dx.doi. org/10.21037/jtd-20-2779

Data Sharing Statement: Available at http://dx.doi. org/10.21037/jtd-20-2779

Conflicts of Interest: All authors have completed the ICMJE uniform disclosure form (available at http://dx.doi. org/10.21037/jtd-20-2779). The authors have no conflicts of interest to declare.

Ethical Statement: The authors are accountable for all aspects of the work in ensuring that questions related to the accuracy or integrity of any part of the work are appropriately investigated and resolved. The study was conducted in accordance with the Declaration of Helsinki (as revised in 2013). The study was approved by The Institutional Review Board of Fuwai Hospital (NO.20201315 ) and informed consent was taken from all the patients.

Open Access Statement: This is an Open Access article distributed in accordance with the Creative Commons
Attribution-NonCommercial-NoDerivs 4.0 International License (CC BY-NC-ND 4.0), which permits the noncommercial replication and distribution of the article with the strict proviso that no changes or edits are made and the original work is properly cited (including links to both the formal publication through the relevant DOI and the license). See: https://creativecommons.org/licenses/by-nc-nd/4.0/.

\section{References}

1. Veselka J, Anavekar NS, Charron P. Hypertrophic obstructive cardiomyopathy. Lancet 2017;389:1253-67.

2. Nishimura RA, Seggewiss H, Schaff HV. Hypertrophic obstructive cardiomyopathy: surgical myectomy and septal ablation. Circ Res 2017;121:771-83.

3. Dearani JA. Septal myectomy remains the gold standard Wigle ED, Rozenblyum E, Fedwick K, Eur Heart J 2012;33:1999-2000.

4. Ommen SR, Maron BJ, Olivotto I, et al. Long-term effects of surgical septal myectomy on survival in patients with obstructive hypertrophic cardiomyopathy. J Am Coll Cardiol 2005;46:470-6.

5. Woo A, Williams WG, Choi R, et al. Clinical and echocardiographic determinants of long-term survival after surgical myectomy in obstructive hypertrophic cardiomyopathy. Circulation 2005;111:2033-41.

6. Wang S, Cui H, Yu Q, et al. Excision of anomalous muscle bundles as an important addition to extended septal myectomy for treatment of left ventricular outflow tract obstruction. J Thorac Cardiovasc Surg 2016;152:461-8.

7. Spirito P, Rossi J, Maron BJ. Alcohol septal ablation: in which patients and why? Ann Cardiothorac Surg 2017;6:369-75.

8. Valeti US, Nishimura RA, Holmes DR, et al. Comparison of surgical septal myectomy and alcohol septal ablation with cardiac magnetic resonance imaging in patients with hypertrophic obstructive cardiomyopathy. J Am Coll Cardiol 2007;49:350-7.

9. Quintana E, Sabate-Rotes A, Maleszewski JJ, et al. Septal myectomy after failed alcohol ablation: Does previous percutaneous intervention compromise outcomes of myectomy? J Thorac Cardiovasc Surg 2015;150:159-67.e1.

10. Faber L, Seggewiss H, Gleichmann U. Percutaneous transluminal septal myocardial ablation in hypertrophic 
obstructive cardiomyopathy: results with respect to intraprocedural myocardial contrast echocardiography. Circulation 1998;98:2415.

11. Nagueh SF, Lakkis NM, He ZX, et al. Role of myocardial contrast echocardiography during nonsurgical septal reduction therapy for hypertrophic obstructive cardiomyopathy. J Am Coll Cardiol 1998;32:225.

12. Suna $S$, Nishino $M$, Sawada T, et al. Intravenous myocardial contrast echocardiography can predict recurrence of pressure gradient of left ventricular outflow tract in hypertrophic obstructive cardiomyopathy after percutaneous transluminal septal myocardial ablation. J Cardiol Cases 2009;1:e25-7.

13. Cui H, Schaff HV, Nishimura RA, et al. Conduction Abnormalities and Long-Term Mortality Following Septal Myectomy in Patients With Obstructive Hypertrophic Cardiomyopathy. J Am Coll Cardiol 2019;74:645-55.

14. Panaich SS, Badheka AO, Chothani A, et al. Results of ventricular septal myectomy and hypertrophic cardiomyopathy (from Nationwide Inpatient Sample [1998-2010]). Am J Cardiol 2014;114:1390-5.

15. Nagueh SF, Buergler JM, Quinones MA, et al. Outcome of surgical myectomy after unsuccessful alcohol septal ablation for the treatment of patients with hypertrophic obstructive cardiomyopathy. J Am Coll Cardiol 2007;50:795-8.

16. Maron BJ, Yacoub M, Dearani JA. Controversies in cardiovascular medicine. Benefits of surgery in obstructive hypertrophic cardiomyopathy: bring septal myectomy back for European patients. Eur Heart J 2011;32:1055-8.

17. Criley JM. Unobstructed thinking (and terminology) is called for in the understanding and management of hypertrophic cardiomyopathy.J Am Coll Cardiol 1997;29:741-3.

18. Quintana E, Bajona P, Arguis MJ, et al. Septal myectomy after failed septal alcohol ablation. Ann Cardiothorac Surg 2017;6:394-401.

19. Fitzgerald P, Kusumoto F. The effects of septal myectomy and alcohol septal ablation for hypertrophic cardiomyopathy on the cardiac conduction system. J Interv Card Electrophysiol 2018;52:403-8.

20. Axelsson A, Weibring K, Havndrup O, et al. Atrioventricular conduction after alcohol septal ablation for obstructive hypertrophic cardiomyopathy. J Cardiovasc Med (Hagerstown) 2014;15:214-21.
21. Chen AA, Palacios IF, Mela T, et al. Acute predictors of subacute complete heart block after alcohol septal ablation for obstructive hypertrophic cardiomyopathy. Am J Cardiol 2006;97:264.

22. Liebregts M, Vriesendorp PA, Ten Berg JM. Alcohol Septal Ablation for Obstructive Hypertrophic Cardiomyopathy: A Word of Endorsement. J Am Coll Cardiol 2017;70:481-8.

23. Alam M, Dokainish H, Lakkis N. Alcohol septal ablation for hypertrophic obstructive cardiomyopathy: a systematic review of published studies. J Interv Cardiol 2006;19:319.

24. Batzner A, Pfeiffer B, Neugebauer A, et al. Survival After Alcohol Septal Ablation in Patients With Hypertrophic Obstructive Cardiomyopathy. J Am Coll Cardiol 2018;72:3087.

25. Chang SM, Nagueh SF, Spencer WH 3rd, et al. Complete heart block: determinants and clinical impact in patients with hypertrophic obstructive cardiomyopathy undergoing nonsurgical septal reduction therapy. J Am Coll Cardiol 2003;42:296.

26. Sorajja P, Ommen SR, Holmes DR Jr, et al. Survival after alcohol septal ablation for obstructive hypertrophic cardiomyopathy. Circulation 2012;126:2374.

27. Vriesendorp PA, Liebregts M, Steggerda RC, et al. Longterm outcomes after medical and invasive treatment in patients with hypertrophic cardiomyopathy. JACC Heart Fail 2014;2:630.

28. Steggerda RC, Damman K, Balt JC, et al. Periprocedural complications and long-term outcome after alcohol septal ablation versus surgical myectomy in hypertrophic obstructive cardiomyopathy: a single-center experience. JACC Cardiovasc Interv 2014;7:1227.

29. Alam M, Dokainish H, Lakkis NM. Hypertrophic obstructive cardiomyopathy-alcohol septal ablation vs. myectomy: a meta-analysis. Eur Heart J 2009;30:1080.

30. Nguyen A, Schaff HV, Hang D, et al. Surgical myectomy versus alcohol septal ablation for obstructive hypertrophic cardiomyopathy: A propensity score-matched cohort. J Thorac Cardiovasc Surg 2019;157:306.

31. Kimmelstiel C, Zisa DC, Kuttab JS, et al. Guideline-Based Referral for Septal Reduction Therapy in Obstructive Hypertrophic Cardiomyopathy Is Associated With Excellent Clinical Outcomes. Circ Cardiovasc Interv 2019;12:e07673.

32. ten Cate FJ, Soliman OI, Michels M, et al. Long-term outcome of alcohol septal ablation in patients with 
obstructive hypertrophic cardiomyopathy: a word of caution. Circ Heart Fail 2010;3:362.

33. Kim LK, Swaminathan RV, Looser P, et al. Hospital Volume Outcomes After Septal Myectomy and

Cite this article as: Yang Q, Zhu C, Cui H, Tang B, Wang S, Yu Q, Zhao S, Song Y, Wang S. Surgical septal myectomy outcome for obstructive hypertrophic cardiomyopathy after alcohol septal ablation. J Thorac Dis 2021;13(2):1055-1065. doi: 10.21037/ jtd-20-2779
Alcohol Septal Ablation for Treatment of Obstructive Hypertrophic Cardiomyopathy: US Nationwide Inpatient Database 2003-2011. JAMA Cardiol 2016;1:324-32. 